\title{
Driving abstinence is necessary after lumbar spinal fusion: a prospective cohort study
}

\author{
Michael C. Liebensteiner ${ }^{1}$ - Franz Birkfellner ${ }^{2} \cdot$ Martina Deibl $^{3} \cdot$ Christian Haid $^{1}$. \\ Martin Krismer $^{1} \cdot$ Dietmar Dammerer $^{1}$
}

Received: 16 March 2016 / Accepted: 27 March 2016/Published online: 22 April 2016

(C) The Author(s) 2016. This article is published with open access at Springerlink.com

\begin{abstract}
Purpose Studies on driving safety after lumbar spinal procedures are rare. Previous studies solely reported on a) driving reaction time (DRT) after lumbar nerve root blocks, b) DRT after discectomy and c) preliminary DRT findings after lumbar fusion.

Methods DRT was assessed with a driving simulator as described before. Measurements were done one day before surgery (preop DRT), one week after surgery (postop1 DRT), three months (postop2 DRT) and one year postoperatively (postop3 DRT). Back pain was determined with visual analogue scales (VAS) on all four occasions. Additionally, we monitored each patient's pre-operative driving frequency and
\end{abstract}

Dietmar Dammerer

dietmar.dammerer@tirol-kliniken.at

Michael C. Liebensteiner

michael.liebensteiner@i-med.ac.at

Franz Birkfellner

franz.birkfellner@khsj.at

Martina Deibl

martina.deibl@i-med.ac.at

Christian Haid

christian.haid@i-med.ac.at

Martin Krismer

martin.krismer@tirol-kliniken.at

1 Department of Orthopaedics, Medical University of Innsbruck, Anichstr. 35, A-6020 Innsbruck, Austria

2 Department of Surgery, Regional Hospital St. Johann, Bahnhofstraße 14, 6380 St. Johann in Tirol, Austria

3 Department of Medical Statistics, Informatics and Health Economics, Medical University of Innsbruck, Anichstr. 35,

A-6020 Innsbruck, Austria intake of analgesics. For statistical analysis we used an ANOVA for repeated measurements.

Results Thirt eight of 51 patients completed all measurements (17 monosegmental fusion, 14 polysegmental fusion, seven other lumbar fusion procedures). The longitudinal changes in DRT showed overall significance $(p=0.013)$. Post-hoc tests determined $p=0.035$ for the DRT-increase from pre- to postoperative. We did not determine a significant statistical effect for the type of surgery $(p=0.581)$ or patient age $(p=0.134)$. A tendency towards statistical significance was ascertained for the influence of patients' driving frequency on DRT $(\mathrm{p}=0.051)$.

Conclusions We found increased DRT at the time of discharge after lumbar spinal fusion and therefore recommend driving abstinence for the time thereafter. Based on our findings it appears safe to return to driving at 3 months postoperative.

Keywords Brake response time - Driving ability - Driving reaction $\cdot$ Driving safety $\cdot$ Lower back pain $\cdot$ Lumbar fusion

\section{Introduction}

Driving ability can be crucial for an individual's participation in social life and for dealing with the daily to-dos. Therefore, the key parameter of driving ability, the 'driving reaction time (DRT), ' has been studied following several common orthopedic treatments like nerve root block [1], total hip arthroplasty (THA) [2, 3], anterior cruciate ligament reconstruction [4, 5], various types of arthroscopic knee procedures [6] and total knee arthroplasty (TKA) [7-9]. Additionally, we recently presented the short-term post-operative driving reaction time (DRT) after primary fusion of the lumbar spine [10]. Although that study contributed new scientific knowledge, it had several shortcomings: the relatively short post-operative 
follow-up period, a small and inhomogeneous patient population and the inability to study the effect of different types of fusion surgery.

We therefore decided to continue our study to further investigate the effect of lumbar fusion on DRT and address the above-mentioned shortcomings on the basis of the following hypotheses.

We hypothesized that DRT shows significant differences in a longitudinal comparison from pre-operative to several occasions during the first post-operative year (hypothesis 1). We further hypothesized that DRT differs significantly for different types of lumbar fusion (hypothesis 2). As further hypotheses we speculated whether driving frequency, age or back pain would influence DRT (hypotheses 3, 4 and 5, respectively).

\section{Materials and methods}

The local ethics committee approved the study protocol and written informed content was obtained from all patients before participation.

Consecutive patients on the operation list awaiting primary fusion of the lumbar spine, between a time interval of 15 months, were considered for inclusion in the study. Of 141 targeted patients, 28 had no driving license, 25 had their surgery date changed or cancelled, 20 could not be contacted due to conflicting investigator schedules and 13 patients refused to participate in the study. Two patients were not approved for surgery by their anesthesiologist or internist and thus could not be operated and two patients wanted to participate but could not sit in our driving simulator. Six patients underwent the preoperative measurement, but dropped out during the postoperative measurements for organizational reasons. Two patients dropped out at the postoperative test date because of pain related to the test procedure. Five patients had to undergo revision surgery (one screw malposition, 0one 00000000 screw-bar disconnection, two removal of hematoma, one dura repair) and were therefore excluded (Fig. 1).

Of the remaining 38 patients 17 underwent monosegmental instrumented posterolateral fusion, 14 underwent polysegmental instrumented posterolateral fusion and seven had other procedures (anterior lumbar interbody fusion or circumferential fusion). The groups were named 'monosegmental', 'polysegmental' and 'other,' respectively. Patients with neurologic deficits were not included. The study protocol was approved by the university's ethics committee, and written informed consent was obtained from all subjects prior to participation. All data were collected prospectively.

The following test procedure was identical to that previously published [10]. DRT was measured with an experimental apparatus based on those previously described and validated in the published literature [1, 2]: An original adjustable car seat was fixed on a frame with hanging pedals mounted on rubber damped pivots. The seat was adjusted to simulate the patient's driving position with regard to seat inclination, head rest and seat-pedal distance, as suggested by previous research [11]. An external suitcase containing the logic gate electronics, a green and a red lamp, was positioned on a table at a constant distance in front of the frame. When the accelerator was fully depressed the green lamp lit up, indicating that the patient was not driving in a 'ready-to-brake fashion.' After an interval of 5 to 10 seconds, the investigator pressed an external trigger concealed from the patient's view, which activated the red lamp and the electronic clock. The subjects were instructed to apply the brake as quickly as possible with their right foot when the signal appeared. The time interval until the subject operated the brake was measured and displayed in milliseconds on the digital clock. The time interval was taken as the DRT. Tests were always performed with the right leg while the left foot rested on the coupler pedal. DRT was measured with this procedure ten times after the subject had been familiarized with the apparatus (three trial runs). An interval of 5 seconds was maintained between measurements. All participants were given the same standardized instructions. Reproducibility tests were deemed unnecessary because we adhered to previous protocols [2]. For each test series, the two shortest and two longest reaction times were discarded. The mean of the remaining six values was taken as the individual's DRT for the respective test date. Tests were carried out one day before surgery (preop DRT), one week after surgery (postop1 DRT), three months (postop2 DRT) and one year after surgery (postop3 DRT).

To assess each patient's intensity of lower back pain we applied visual analogue scales (VAS) on all four test dates. Additionally, each participant had to check one of the following items with regard to his/her driving frequency: never, seldom, sometimes, often, very often. The driving frequency data was only obtained pre-operatively. We also assessed the intake of analgesics on all four test dates according to the threestep pain ladder of the World Health Organization [12].

Data were further processed using the Statistical Package for Social Sciences (SPSS-Norusis/SPSS Inc., Chicago, IL, USA). Before the study was initiated in 2007 we performed a case rate estimation for hypothesis 1. Because no previous studies on driving ability after lumbar fusion were available, we utilized the DRT data published for total hip arthroplasty [3]. The authors reported a DRT of $704( \pm 39)$ msec pre-operatively and $591( \pm 40) \mathrm{msec}$ post-operatively. On this basis we determined an effect size of 2.86. Assuming alpha $=0.017$ (see below) and beta $=0.2$ we calculated that five patients 
Fig. 1 Flowchart for patients enrolled in study
141 patients identified on the operation list

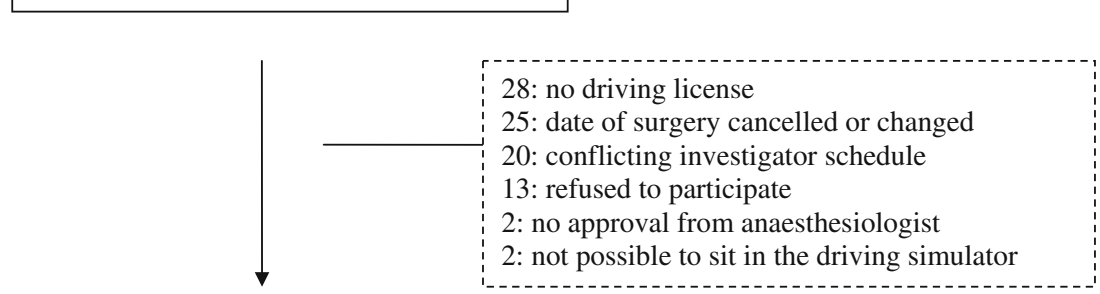

51 patients included in the study

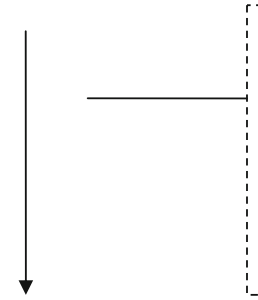

13 drop outs

- 5 excluded due to revision surgery

- 8 excluded due to incomplete postoperative data

○ 6: organizational reasons

- 2: pain related to the test procedure would be necessary to confirm or reject hypothesis 1 . Because we doubted the small variance and because we additionally intended to investigate for group differences we decided to include at least 30 patients in the study. The data sets were determined to be normally distributed by means of Kolmogorov-Smirnov tests. Analysis of variance for repeated measurements (general linear model) was performed, with DRT as dependent variable and time as a factor. Statistical significance was defined as $p<0.05$. In the case of significant overall influence of time on DRT, we performed a post-hoc $t$ test for dependent variables at an alpha level of 0.017 for the following pairs of variables: DRT preop - DRT postop1, DRT preop - DRT postop2, DRT preop - DRT postop3. We then tested for inter-subject effects to determine the influence of age and driving frequency. Pearson correlation coefficients were calculated to test for an association between VAS back pain and DRT.

\section{Results}

Participant characteristics are provided in Table 1. Preoperatively, DRT was $693( \pm 142)$ milliseconds $(\mathrm{msec})$ and increased to 748 $( \pm 224)$ msec at the postop1 measurement. At the postop2 measurement DRT decreased to $657( \pm 118) \mathrm{msec}$ and then slightly increased to $671( \pm 171) \mathrm{msec}$ at the postop3 measurement at one year. We determined statistical overall significance for these longitudinal differences $(\mathrm{p}=0.013)$ (hypothesis 1$)$. Post-hoc tests (alpha $=0.017)$ revealed a statistical tendency for the DRTworsening from 'preop DRT' to 'postop1 DRT' $(p=0.035)$. A statistical trend was also determined for the comparison 'preop DRT and postop2 DRT' ( $p=0.019$ ) (Fig. 2).

Descriptive statistics of the DRT values achieved by the various surgical groups are presented in Table 2. Although a trend towards slightly longer DRT values is shown in the polysegmental group, we did not determine a significant statistical effect of the surgical group ( $\mathrm{p}=0.581$; hypothesis 2 ). Patients rated their driving frequency as follows: never: 3 , seldom: 3 , sometimes: 8 , often: 11 , very often 13 . A tendency towards statistical significance was ascertained for the influence of patients' driving frequency ( $\mathrm{p}=0.051$; hypothesis 3 ). Age was found to not influence DRT on any of the test dates $(\mathrm{p}=0.134$; hypothesis 4$)$. Moderate and positive correlation coefficients were calculated for VAS back pain and DRT on several test dates (Table 3; hypothesis 5).

\section{Discussion}

The most important finding of our study was the postoperative increase of DRT 1 week after surgery. Given the increase in the descriptive values for DRT from pre- to postoperative and the associated statistical trend towards significance we regard

Table 1 Participant characteristics with regard to age and gender

\begin{tabular}{lllll}
\hline & Monosegmental & Polysegmental & Other & $\mathrm{p}$ value \\
\hline Age $[\mathrm{y}](\mu \pm \mathrm{sd})$ & 49 & 57 & 49 & 0.075 \\
Male [n] & 3 & 7 & 2 & 0.153 \\
Female [n] & 14 & 7 & 5 & \\
\hline
\end{tabular}


Fig. 2 Longitudinal DRT characteristics of all patients

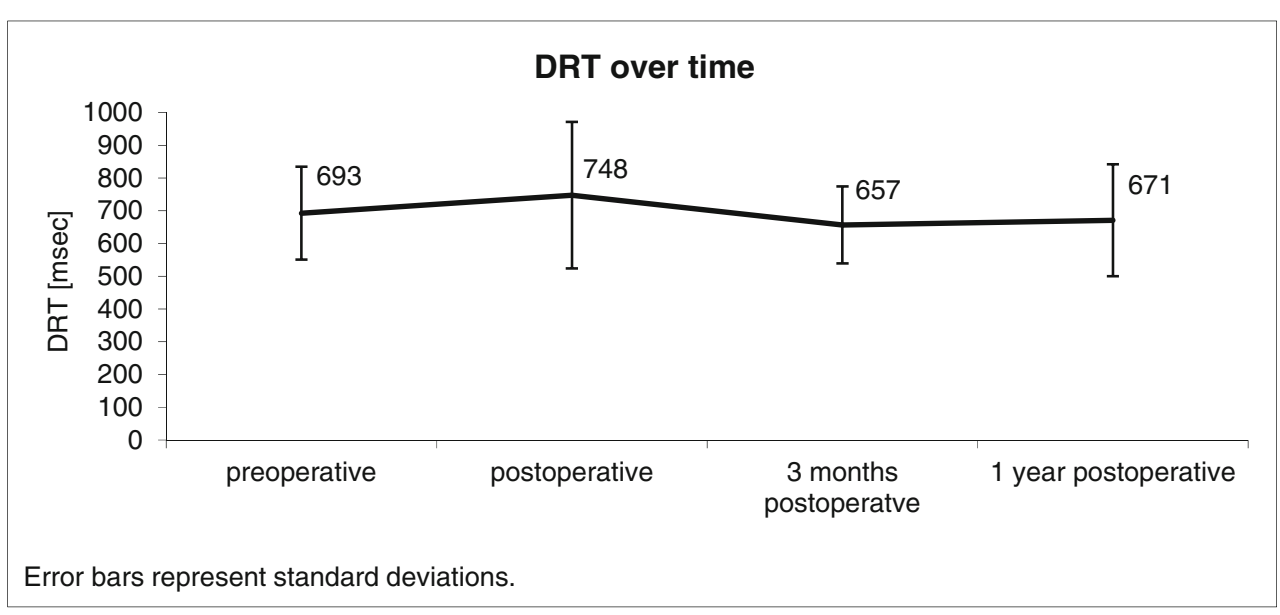

the changes as clinically relevant and the statistics as suffering from type 2 error. Therefore, it seems wise to meanwhile recommend driving abstinence after discharge because of safety reasons. This is in contrast to what was previously published [10]. From one week postoperative to three months postoperative the DRT decreased and was even better than the preoperative reference value ('statistical tendency', $\mathrm{p}=0.019$ ). The DRT value at one year postoperative $(671 \mathrm{msec})$ showed a slight drop in comparison to the DRT at three months, but is still better than the DRT at one week postoperative (Fig. 2). Unfortunately, we did not collect DRT data between 1 week and 3 months postoperative. Consequently, it is not possible to determine the precise moment of DRT recovery after lumbar fusion. After taking all four DRT measurements together, we feel it is necessary to advise patients to refrain from driving for the first 3 postoperative months.

The relationship between orthopedic spinal interventions and driving ability has only rarely been investigated before $[1,10,13]$. Al-Khayer et al. investigated DRT in the context of radiculopathy and nerve root blocks [1]. They reported DRT to be increased two weeks after lumbar nerve root blocks. After six weeks the differences in DRT were no longer

Table 2 Descriptive statistics for DRT dependent on the surgical procedure

\begin{tabular}{llll}
\hline$[\mathrm{msec}]$ & Moneonosegmental & Polysegmental & Other \\
\hline DRT preop & $697( \pm 138)$ & $702( \pm 166)$ & $663( \pm 108)$ \\
DRT 1 week & $731( \pm 268)$ & $773( \pm 211)$ & $739( \pm 138)$ \\
DRT 3 months & $657( \pm 108)$ & $680( \pm 149)$ & $607( \pm 48)$ \\
DRT 1 year & $627( \pm 45)$ & $733( \pm 260)$ & $653( \pm 115)$ \\
\hline
\end{tabular}

DRT: driving reaction time

Monosegmental: monosegmental primary instrumented posterolateral fusion

polysegmental: polysegmental primary instrumented posterolateral fusion

other: other technique for primary fusion of the spine significant. Al-Khayer et al. did not give clear recommendations on when patients may resume driving. Moreover, it seems difficult to directly compare our findings with that study, because the significant postoperative impairment of DRT reported therein might have resulted from direct administration of local anesthetics to the nerve root. Thaler et al. [13] reported on DRT in patients who underwent surgery for lumbar disc herniation and found no worsening of DRT at the time of discharge. Therefore, they did not recommend driving abstinence at all. In a preliminary study our study group already investigated DRT in the context of lumbar spinal fusion [10]. Due to the limitations of that study we did not even find a statistical tendency for postoperative worsening of the DRT and recommended no driving abstinence after discharge.

DRT has been investigated in the context of other orthopedic procedures such as total hip arthroplasty [2, 3], total knee arthroplasty [7, 8], anterior cruciate ligament reconstruction [5] and different types of arthroscopic knee procedures [6]. Based on impaired DRT findings those publications recommended driving abstinences of one to eight weeks, depending on the type of treatment. We assume that procedures

Table 3 Pearson correlation coefficients for VAS back pain and DRT on the four test dates

\begin{tabular}{clllll}
\hline & $\begin{array}{l}\text { DRT } \\
\text { preop }\end{array}$ & $\begin{array}{l}\text { DRT } \\
\text { 1 week }\end{array}$ & $\begin{array}{l}\text { DRT } \\
\text { 3 months }\end{array}$ & $\begin{array}{l}\text { DRT } \\
\text { 1 year }\end{array}$ \\
\hline VAS preop & $\mathrm{r}$ & $0.364^{*}$ & & & \\
& $\mathrm{p}$ & 0.024 & & & \\
VAS 1 week & $\mathrm{r}$ & 0.203 & & \\
& $\mathrm{p}$ & 0.221 & & \\
VAS & $\mathrm{r}$ & & $0.371^{*}$ & \\
3 months & $\mathrm{p}$ & & 0.022 & $0.424^{* *}$ \\
VAS 1 year & $\mathrm{r}$ & & & 0.008 \\
& $\mathrm{p}$ & & & \\
\hline
\end{tabular}

$r$ : Pearson correlation coefficient, $p$ : $\mathrm{p}$ value; VAS: visual analogue scale back pain; DRT: driving reaction time; preop: pre-operatively 
performed in the lower limbs more directly affect DRT via foot transfer time by inducing pain and muscular reflex inhibition.

Despite the fact that a slightly longer DRT in our polysegmental group was indicated by the descriptive statistics (Table 2), we found no statistical significance for the surgical group as a factor $(\mathrm{p}=0.581)$. The patients in our polysegmental group underwent fusion of 2.6 segments on average (range 24). We therefore believe our recommendations are valid for the majority of types of lumbar fusion. Our findings suggest that DRT is probably influenced by a patient's driving frequency (statistical tendency, $\mathrm{p}=0.051$ for driving frequency as influencing factor). However, our findings did not achieve statistical significance. Previous studies found better DRT after total knee arthroplasty in patients with more driving experience [7]. Therefore, we assume that DRT after spinal fusion could also be related to driving frequency and recommend that this question be investigated in future studies.

With regard to our hypothesis 4 we did not observe a significant statistical effect of age on DRT after spinal fusion. Other researchers investigated other orthopedic procedures and did not report associations between DRT and age $[2,7,14,15]$. The current study assessed significant associations between VAS pain and DRT on several test dates. These findings are partly contrasted [1] and partly supported by previous studies [10]. As potential pathomechanism, pain could have induced reflex inhibition of muscles and thus increased foot transfer time. Interestingly, the first postoperative test revealed no correlations between VAS pain and DRT. We speculate whether this can be explained by the administration of relatively higher doses of analgesic medication immediately postoperative.

This study is important for driving safety in that it overrules the findings and recommendations of our previous study. However, we acknowledge the following limitations: We did not collect DRT data between 1 week and 3 weeks postoperative. In addition, the postoperative increase in DRT was statistically just a trend (possibly type 2 error). Moreover, DRT is an important factor of driving ability but not the only one.

We found increased DRT at the time of discharge after lumbar spinal fusion and therefore recommend driving abstinence for the time thereafter. Based on our findings it appears safe to return to driving at 3 months postoperative. These findings are applicable regardless of which type of spinal fusion was performed.

Acknowledgments Open access funding provided by University of Innsbruck and Medical University of Innsbruck.

\section{Compliance with ethical standards}

Conflict of Interest No benefits in any form have been received or will be received from a commercial party related directly or indirectly to the subject of this article.
The authors did not receive any outside funding or grants in support of their research for or preparation of this work. Neither they nor a member of their immediate families received payments or other benefits or a commitment or agreement to provide such benefits from a commercial entity. No commercial entity paid or directed, or agreed to pay or direct, any benefits to any research fund, foundation, division, center clinical practice, or other charitable or nonprofit organization with which the authors, or a member of their immediate families, are affiliated or associated.

Open Access This article is distributed under the terms of the Creative Commons Attribution 4.0 International License (http:// creativecommons.org/licenses/by/4.0/), which permits unrestricted use, distribution, and reproduction in any medium, provided you give appropriate credit to the original author(s) and the source, provide a link to the Creative Commons license, and indicate if changes were made.

\section{References}

1. Al-khayer A, Schueler A, Kruszewski G, Armstrong G, Grevitt MP (2008) Driver reaction time before and after treatment for lumbar radiculopathy. Spine 33:1696-1700

2. Ganz SB, Levin AZ, Peterson MG, Ranawat CS (2003) Improvement in driving reaction time after total hip arthroplasty. Clin Orthop Relat Res 413:192-200

3. MacDonald W, Owen JW (1988) The effect of total hip replacement on driving reactions. J Bone Joint Surg (Br) 70:202-205

4. Gotlin RS, Sherman AL, Sierra N, Kelly M, Scott WN (2000) Measurement of brake response time after right anterior cruciate ligament reconstruction. Arthroscopy 16:151-155

5. Gotlin RS, Sherman AL, Sierra N, Kelly MA, Pappas Z, Scott WN (2000) Measurement of brake response time after right anterior cruciate ligament reconstruction. Arch Phys Med Rehabil 81:201-204

6. Hau R, Csongvay S, Bartlett J (2000) Driving reaction time after right knee arthroscopy. Knee Surg Sports Traumatol Arthrosc 8:89-92

7. Spalding TJ, Kiss J, Kyberd P, Turner-Smith A, Simpson AH (1994) Driver reaction times after total knee replacement. J Bone Joint Surg (Br) 76:754-756

8. Liebensteiner MC, Kern M, Haid C, Kobel C, Niederseer D, Krismer M (2010) Brake response time before and after total knee arthroplasty: a prospective cohort study. BMC Musculoskelet Disord 11:267

9. Marques CJ, Barreiros J, Cabri J, Carita AI, Friesecke C, Loehr JF (2008) Does the brake response time of the right leg change after left total knee arthroplasty? A prospective study. Knee 15:295-298

10. Liebensteiner MC, Birkfellner F, Thaler M, Haid C, Bach C, Krismer M (2010) Driving reaction time before and after primary fusion of the lumbar spine. Spine 35:330-335

11. Scott PA, Candler PD, Li JC (1996) Stature and seat position as factors affecting fractionated response time in motor vehicle drivers. Appl Ergon 27:411-416

12. The pain ladder of the WHO. World Health Organization

13. Thaler M, Lechner R, Foedinger B, Haid C, Kavakebi P, Galiano K et al (2012) Driving reaction time before and after surgery for lumbar disc herniation in patients with radiculopathy. Eur Spine J 21: 2259-2264

14. Marques CJ, Cabri J, Barreiros J, Carita AI, Friesecke C, Loehr JF (2008) The effects of task complexity on brake response time before and after primary right total knee arthroplasty. Arch Phys Med Rehabil 89:851-855

15. Pierson JL, Earles DR, Wood K (2003) Brake response time after total knee arthroplasty: when is it safe for patients to drive? J Arthroplasty 18:840-843 